\title{
Hybridization of Localized and Guided Modes in 2D Metal- Insulator-Metal Nanocavity Arrays
}

\author{
Wei Zhou, ${ }^{\dagger}$ Jae Yong Suh, ${ }^{\ddagger}$ Yi Hua, ${ }^{\dagger}$ and Teri W. Odom ${ }^{*} \dagger, \ddagger$ \\ ${ }^{\dagger}$ Department of Materials Science and Engineering and ${ }^{\ddagger}$ Department of Chemistry, Northwestern University, Evanston, Illinois \\ 60208, United States
}

\section{Supporting Information}

ABSTRACT: This article describes the angle-dependent optical responses of $2 \mathrm{D}$ metal-insulator-metal (MIM) nanocavity arrays. Through a combination of soft nanolithography and template stripping, we fabricated arrays of plasmonic MIM nanostructures with subwavelength spacings over square centimeter areas. We controlled the coupling between the localized surface plasmon and guided modes as well as engineered the optical band structure by tuning the insulator thickness. Rabi splitting of hybridized modes strongly depended on the spatial overlap of the near-fields of the localized and guided modes.

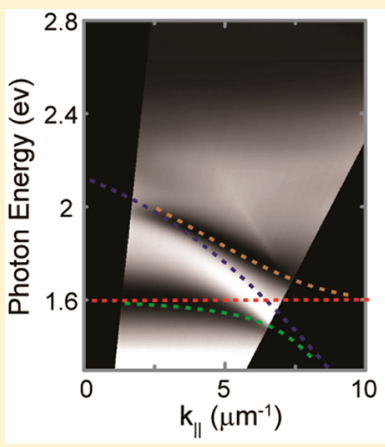

\section{INTRODUCTION}

Large arrays of periodic metal nanostructures (plasmonic crystals) can support both localized and delocalized surface plasmons (SPs). ${ }^{1,2}$ The ability of plasmonic crystals to concentrate and manipulate optical energy on subwavelength scales is important for a range of applications such as chemical and biological sensing, photovoltaics, optoelectronics, and nonlinear optics. ${ }^{3-10}$ Compared with typical plasmonic crystals that are periodically nanotextured at a continuous metalinsulator interface, ${ }^{11}$ arrays of metal-insulator-metal (MIM) multilayer structures can support a richer set of optical properties because of the interplay between SP modes at different metal-insulator interfaces. ${ }^{12,13}$ For example, MIM plasmonic nanocavity arrays have shown negative refractive index, Fano resonances, electromagnetic-induced transparency, and broadband optical absorption. ${ }^{14-16}$

One-dimensional arrays of MIM nanocavities have been extensively investigated; ${ }^{13-15}$ however, the 1D translational symmetry imposes restrictions on allowed excitation polarizations and directions. Two-dimensional arrays of MIM plasmonic nanocavities not only avoid such constraints but also support optical responses that can be tailored by additional design parameters (e.g., lattice symmetry and nanostructure shape). ${ }^{16-20}$ Because of sample fabrication challenges, most work on 2D arrays of MIM nanocavities has focused on the optical properties at normal incident angles, ${ }^{16-20}$ which has precluded characterization of their optical band structure. Although a few papers have investigated the angular responses of MIM nanocavity arrays, ${ }^{21,22}$ how spatial dispersion affects coupling between localized and delocalized modes is not known.

Here we show that the optical band structure of 2D MIM plasmonic nanocavity arrays can be significantly modified by strong coupling between localized and guided SP modes. In the angle-resolved optical measurements, we observed anticrossing behavior between localized surface plasmon (LSP) and guided modes that showed a large Rabi splitting energy $(0.22 \mathrm{eV})$. Numerical simulations revealed that new hybridized states were formed with different resonant energies and different local field distributions because of interference between the LSP and guided modes. Also, as the insulator layer thickness increased, MIM nanocavity arrays supported higher order guided modes with transverse magnetic (TM) and transverse electric (TE) character. Compared with TM guided modes, TE modes exhibited a much larger Rabi splitting energy when coupled to localized modes because of increased overlap between the local optical fields.

\section{EXPERIMENTAL METHODS}

Large-area $\left(>1 \mathrm{~cm}^{2}\right)$ arrays of 2D MIM nanocavities were produced with different insulator layer thicknesses $t$. Figure 1A summarizes the major steps in the fabrication process. First, ultrasmooth $\mathrm{Au}$ surfaces were created by template-stripping $\mathrm{Au}$ films (thickness $=100 \mathrm{~nm}$ ) on $\mathrm{Si}(100)$ wafers using a polyurethane (PU) bonding layer. ${ }^{2}$ This preparation of ultraflat $\mathrm{Au}$ films was necessary to reduce scattering losses of plasmon resonances from surface roughness. Second, thin films of poly(methyl methacrylate) (PMMA) with different $t$ were spincoated on the Au film by diluting the concentration of PMMA with chlorobenzene $(0.5-6 \%$, by weight). Finally, Au nanohole

Special Issue: Nanostructured-Enhanced Photoenergy Conversion

Received: July 13, 2012

Revised: August 20, 2012

Published: August 22, 2012 


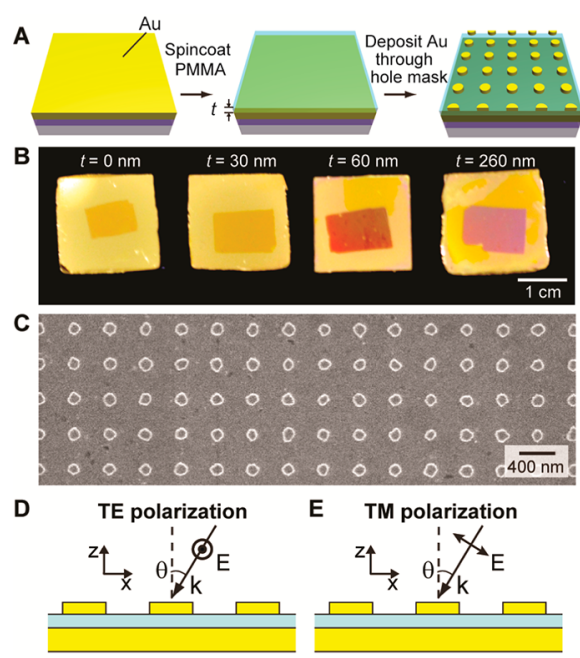

Figure 1. Large-area fabrication of $2 \mathrm{D}$ arrays of MIM plasmonic nanocavities. (A) Fabrication scheme. (B) Optical micrographs of Au NP arrays on Au-PMMA multilayer with different PMMA thicknesses $t$ as indicated (Au NP array: $h=40 \mathrm{~nm}, d=150 \mathrm{~nm}, a_{0}=400 \mathrm{~nm}$ ). (C) SEM image of MIM arrays. (D) TE- and (E) TM-polarized excitation conditions for the angle-resolved optical measurements.

arrays fabricated by PEEL ${ }^{23}$ were used as deposition masks to create periodic arrays of Au NPs (height $h=40 \mathrm{~nm}$, diameter $d$ $=150 \mathrm{~nm}$, periodicity $a_{0}=400 \mathrm{~nm}$ ) directly on the insulating PMMA layer. Optical micrographs of samples with various thicknesses of PMMA $(t=0,30,60$, and $260 \mathrm{~nm})$ showed dramatically different colors (Figure $1 \mathrm{~B})$. The size variation of individual NPs was estimated to be $\sim 20 \%(150 \pm 15 \mathrm{~nm})$ based on scanning electron microscopy (SEM) images (Figure $1 \mathrm{C})$.

Angle-resolved optical measurements were used to measure the optical band structure of 2D MIM nanocavity arrays. The samples were mounted on a computer-controlled stage that could be rotated at a range of incident angles $\left(\theta=10-60^{\circ}\right)$ with $0.02^{\circ}$ accuracy for reflectance measurements. Collimated white light from a $100 \mathrm{~W}$ halogen source was incident on the sample surface, and the reflected light was directed to a CCDspectrometer $\left(\mathrm{LN}_{2}\right.$-cooled $\mathrm{CCD} / \mathrm{Triax} 522$, Horiba Jobin Yvon). Zero-order reflectance spectra were measured under TE- or TM-polarized light (Figures 1D,E), where the $x$ and $y$ axes were along the high-symmetry lattice direction of the square MIM arrays, and the $z$ axis was defined by the surface normal of the sample. We used a linear interpolation algorithm to convert the measured reflectance data (wavelength $\lambda$ vs $\theta$ ) into dispersion diagrams (photon energy $E$ vs in-plane wavevector of light $k_{\|}$).

\section{RESULTS AND DISCUSSION}

As a first-order approximation, we decomposed the optical properties of 2D MIM nanocavity arrays into responses from localized SP modes in individual nanoparticles and delocalized SP slab modes. In a previous work, the delocalized modes of MIM arrays were treated as propagating surface plasmon polaritons (SPPs). ${ }^{18}$ To consider the effects of $t$, however, we propose a more rigorous description to treat them as guided modes in a metal-insulator-air (M-I-A) multilayered system (Figure 2A). Now, SPPs at the metal-insulator interface become generalized as a type of $\mathrm{TM}_{0}$-guided mode when $t=0$. On the basis of Maxwell's equations and appropriate boundary
A

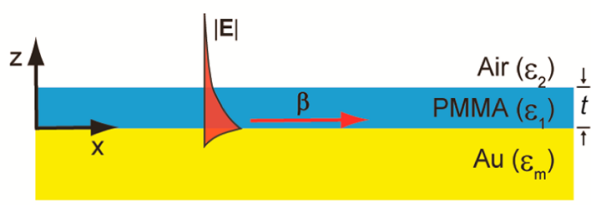

B

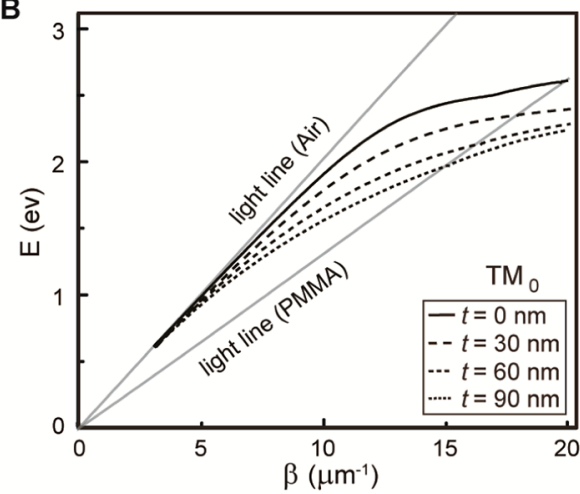

Figure 2. Guided modes in an asymmetric metal-insulator-air planar structure depend on the insulator layer thickness. (A) Scheme of $\mathrm{TM}_{0}$ guided modes in an $\mathrm{M}-\mathrm{I}-\mathrm{A}$ multilayer structure. (B) Calculated $E-\beta$ dispersion curves of the $\mathrm{TM}_{0}$ modes in Au-PMMA-air planar structure with different PMMA thickness $(t=0,30,60$, and $90 \mathrm{~nm})$.

conditions, the energy $(\hbar \omega)$-wavevector $(\beta)$ dispersion relations of TM- and TE-guided modes in a $\mathrm{M}-\mathrm{I}-\mathrm{A}$ structure can be determined as ${ }^{24,25}$

$$
(\mathrm{TM} \text { modes }) \tan \kappa t=\varepsilon_{1} \kappa\left(\varepsilon_{\mathrm{m}} \gamma+\varepsilon_{2} \delta\right) /\left(\varepsilon_{2} \varepsilon_{\mathrm{m}} \kappa^{2}-\varepsilon_{1}^{2} \gamma \delta\right)
$$

$$
\text { (TE modes) } \tan \kappa t=\kappa(\gamma+\delta) /\left(\kappa^{2}-\gamma \delta\right)
$$

where $\varepsilon_{1}, \varepsilon_{2}$, and $\varepsilon_{\mathrm{m}}$ are dielectric constants for PMMA $\left(\varepsilon_{1}\right.$ $=2.16)$, air $\left(\varepsilon_{2}=1\right)$, and Au. The parameters $\kappa, \gamma$, and $\delta$ are $\kappa=$ $\left(\varepsilon_{1} k_{0}^{2}-\beta^{2}\right)^{-1 / 2}, \gamma=\left(\beta^{2}-\varepsilon_{2} k_{0}^{2}\right)^{-1 / 2}$, and $\delta=\left(\beta^{2}-\varepsilon_{\mathrm{m}} k_{0}^{2}\right)^{-1 / 2}$, where $\beta$ is the wavevector of the guided modes and $k_{0}=\omega / c$ is the wavevector of photons in air. Because $\varepsilon_{\mathrm{m}}$ of Au is complex and highly dispersive at optical frequencies, the $E-\beta$ relations of TM or TE modes can only be determined numerically from eqs 1 and 2 . We treated $\varepsilon_{\mathrm{m}}$ of Au by a Lorentz-Drude model with parameters fitted to Johnson and Christy data. ${ }^{26}$

Numerical calculations revealed that only the $\mathrm{TM}_{0}$-guided mode was supported in the M-I-A structure with small insulator thicknesses $(t<100 \mathrm{~nm})$ at optical energies $(1.2 \mathrm{eV}<$ $E<3.1 \mathrm{eV})$. The calculated $E-\beta$ dispersion curves of the $\mathrm{TM}_{0}$ mode were flat-banded at high energies with a decreased group velocity and asymptotically converged to the air light line at low energies (Figure 2B). As $t$ increased from 0 to $90 \mathrm{~nm}$, the dispersion curve of the $\mathrm{TM}_{0}$ mode gradually shifted to lower energies, which indicates that the optical fields are increasingly confined in the PMMA layer. The dispersion curves of the guided modes always lie below the air light line; thus, their coupling with free-space light in air is forbidden because of momentum mismatch $(|\beta|<(\omega / c))$. By adding a periodic array of Au NPs on top of the M-I-A multilayer structure, however, grating vectors can be used to overcome the momentum mismatch. For excitations where the incident plane is along a high-symmetry direction ( $x$ axis), the Bragg coupling condition $\left(|\beta|=\left|\mathbf{k}_{\|}+\mathbf{G}\right|\right)$ can be explicitly expressed as 

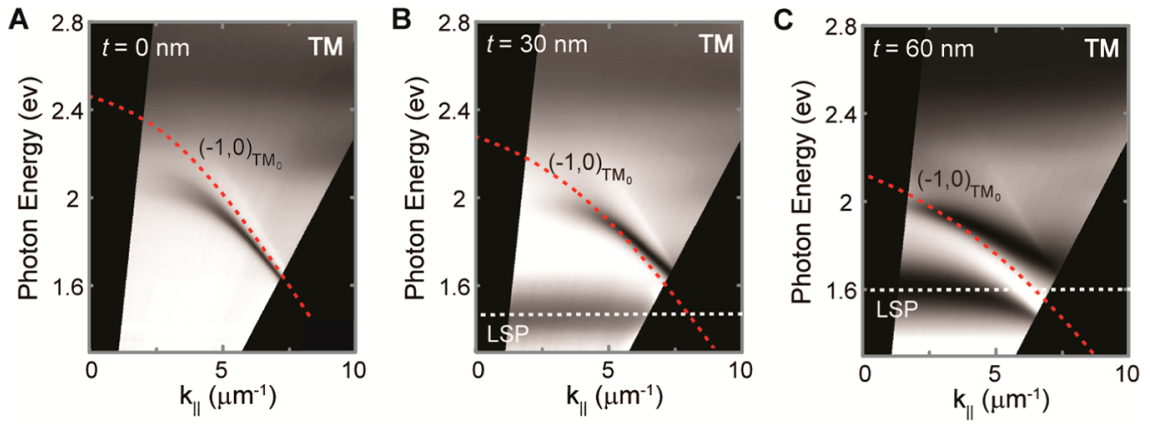

Figure 3. Optical band structure of MIM plasmonic nanocavity arrays depends strongly on the insulator layer thickness. (A-C) Measured reflectance spectra $\left(\theta\right.$ from 0 to $\left.60^{\circ}\right)$ as a function of in-plane wavevector $\left(k_{\|}=k_{0} \cdot \sin \theta\right)$ and energy $(E=\hbar \omega)$ under TM-polarized light for samples with different PMMA layer thicknesses $(t=0,30$, and $60 \mathrm{~nm})$.

$$
|\beta|=\sqrt{\left(k_{\|}+i \frac{2 \pi}{a_{0}}\right)^{2}+\left(j \frac{2 \pi}{a_{0}}\right)^{2}}
$$

$(0.22 \mathrm{eV})$ of hybridized modes ${ }^{1,30,31}$ because of strong coupling when they have the same energy and wavevector.

To understand Rabi splitting between localized and guided modes in MIM nanocavity arrays, we performed 3D finite difference time domain (FDTD) calculations to simulate both far-field and near-field optical responses (FDTD solution, Lumerical, Vancouver, Canada). A single unit cell was modeled with a uniform mesh size of $4 \mathrm{~nm}$ ( $x, y$, and $z$ directions) and with Bloch boundary conditions in $x-y$ dimensions and a perfectly matched layer in $z$. Figure $4 \mathrm{~A}$ displays the FDTD-

guided modes in 2D MIM plasmonic nanocavity arrays.

To discriminate among spectral features in the MIM nanocavity arrays, dispersion diagrams were constructed by displaying spectra as a function of $k_{\|}$and $E$; then, calculated dispersion curves of the guided modes in the $\mathrm{M}-\mathrm{I}-\mathrm{A}$ structure were superimposed (Figure 3). As expected, the interband transition of the Au film was represented by a broad absorption band at $2.5 \mathrm{eV}$, which was independent of $t$ as well as $\theta$ and polarization of the incident light. When Au NP arrays were in contact with the Au surface $(t=0 \mathrm{~nm})$, no plasmonic spectral features were excited under TE polarization (Supporting Information, Figure S1A). In contrast, under TM polarization, a distinct resonance dip started to emerge at $k_{\|}=2.5 \mu \mathrm{m}^{-1}$, which shifted to lower energies as $k_{\|}$increased further (Figure $3 \mathrm{~A})$. The overall shape of this band was in good agreement with the calculated dispersion curve of the $(-1,0) \mathrm{TM}_{0}$ mode. Discrepancy between experiment and theory at low $k_{\|}(<4$ $\mu \mathrm{m}^{-1}$ ) is attributed to the stop band at $k_{\|}=0$ formed by counter-propagating Bloch waves in a periodic structure. ${ }^{27,28}$

After insertion of a PMMA layer $(t=30 \mathrm{~nm})$ between the $\mathrm{Au}$ $\mathrm{NP}$ array and Au film, a broad band appeared at $1.44 \mathrm{eV}$ under both TE and TM polarizations (Figure 3B, Supporting Information, Figure S1B). Polarization and angle-independent optical responses suggest that the broad resonance corresponds to the LSPs of individual NPs. Moreover, the introduction of a PMMA layer results in the red shift of the narrow dispersive band (from 2.05 to $1.95 \mathrm{eV}$ at $k_{\|}=3 \mu \mathrm{m}^{-1}$ ) (Figure 3B), which confirms the calculated effects of $t$ on the $\mathrm{TM}_{0}$ mode (Figure 2B). As $t$ increased from 30 to $60 \mathrm{~nm}$, the energy of the LSP resonance increased from 1.45 to $1.64 \mathrm{eV}$ under TE polarization (Supporting Information, Figure S1C) because of weak coupling with the Au surface. ${ }^{29}$ Under TM polarization, both LSP and guided modes were excited (Figure 3C); however, a simple overlap of the guided (white dashed line) and localized (red dashed line) bands was not present. Instead, as $k_{\|}$increased, the LSP band became narrower and was pushed to lower energies, whereas the guided mode band became broader and was pushed to higher energies. This strong anticrossing behavior can be regarded as a type of Rabi splitting
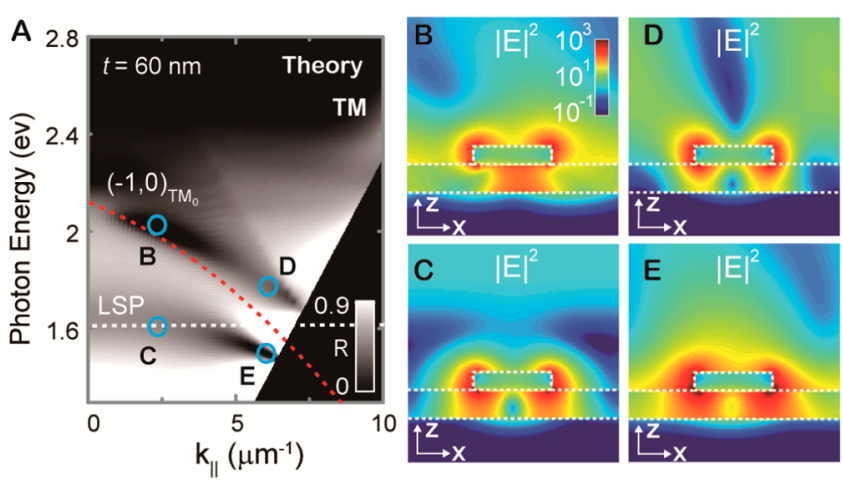

Figure 4. Anticrossing behavior between localized and guided modes arises because of near-field hybridization. (A) FDTD-calculated band diagram of a $2 \mathrm{D}$ array of MIM plasmonic nanocavities $(t=60 \mathrm{~nm})$ under TM-polarized light. (B-E) $|E|^{2}$ distribution maps corresponding to the points $\mathrm{B}-\mathrm{E}$ in the calculated band diagram in panel $\mathrm{A}$.

calculated dispersion diagram of MIM plasmonic nanocavity arrays $(t=60 \mathrm{~nm})$ illuminated by TM-polarized light, which agrees well with measurements in Figure 3C. We calculated and compared the near-field optical properties of the guided and LSP modes from when there was no coupling to when there was hybridization in the anticrossing region at four different points (labeled as $\mathrm{B}-\mathrm{E}$ ).

Figure $4 \mathrm{~B}$ shows that the $(-1,0) \mathrm{TM}_{0}$-guided mode confines intense local fields $\left(|E|^{2} /\left|E_{0}\right|^{2}>200\right)$ uniformly across the entire gap region between the $\mathrm{Au} \mathrm{NP}$ array and the $\mathrm{Au}$ film. Additionally, optical fields with $|E|^{2} /\left|E_{0}\right|^{2}>10$ were induced both inside as well as close to the insulator layer extending over the entire unit cell. Becuase the phase of $E_{z}$ was the same on the top and bottom of the NPs, excitation of the guided mode was accompanied by out-of-plane charge oscillations in individual NPs (Supporting Information, Figure S2A,B). As $\theta$ or $k_{\|}$ decreased, the resonance dips of the guided mode became 
shallower because of the reduced $E_{z}\left(\left|E_{z}\right|=\left|E_{0}\right| \sin \theta\right)$ under TM-polarized excitation. Compared with the $(-1,0) \quad \mathrm{TM}_{0}$ guided mode, the LSP mode had a nearly symmetric profile with large field concentrations $\left(|E|^{2} /\left|E_{0}\right|^{2}>600\right)$ surrounding individual NPs (Figure 4C). In particular, there is a node that appears in the center of the high-field region between the $\mathrm{Au}$ NPs and the Au surface. Correspondingly, the direction of $E_{z}$ is opposite on the two sides of the MIM gap region (Supporting Information, Figure S2C) because of the near-field interactions between individual NPs with their images in the Au surface. ${ }^{29}$ The LSP mode can thus be viewed as a quadrupole plasmon resonance that is equivalent to a pair of coupled dipole oscillators with opposite phase.

Figure 4D,E depicts near-field $|E|^{2}$ distribution maps of the upper and lower energy branches of the hybridized modes when the guided mode has the same energy and wavevector as the LSP mode. In the Rabi splitting region, both branches exhibit mixed near-field character of localized and guided modes: (1) there is a very large local field concentration $\left(|E|^{2} / \mid\right.$ $E_{0} I^{2}>500$ ) in the gap (with a node in the center), and (2) a 10fold higher field enhancement $\left(|E|^{2} /\left|E_{0}\right|^{2}>10\right)$ surrounds the insulator layer across the entire unit cell. Upon closer inspection, we found that the lower energy branch tends to concentrate more optical energy in the high-index insulator layer compared with the upper energy branch. Figure 5

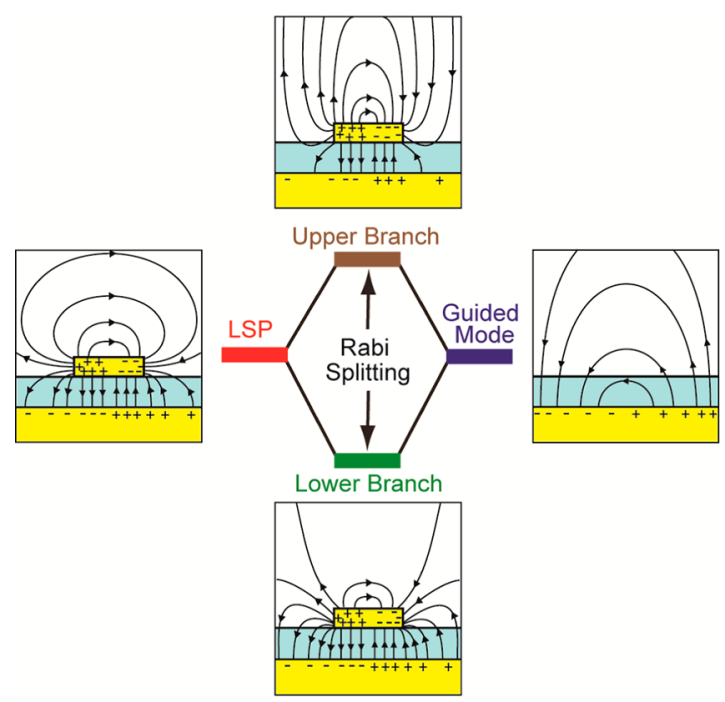

Figure 5. Scheme of Rabi splitting of hybridized modes formed by the LSP band and guided mode. The lower energy hybridized mode concentrates its optical energy in the high- $\varepsilon$ region (insulator layer), whereas the upper energy mode has a larger fraction of energy in the low- $\varepsilon$ region (air).

illustrates that depending on their relative phase difference, interference between the LSP and guided modes can enhance electric fields in either the high- $\varepsilon$ region or the low- $\varepsilon$ region. Therefore, Rabi splitting arises because of the formation of two hybridized modes with different local field distributions.

For a $\mathrm{M}-\mathrm{I}-\mathrm{A}$ multilayer structure with a large PMMA layer thickness $(t \approx 260 \mathrm{~nm})$, eqs 1 and 2 predict that higher order $\mathrm{TE}_{0}$ and $\mathrm{TM}_{1}$ modes emerge with cutoff energies of 1.1 and 1.8 $\mathrm{eV}$, respectively (Figure 6A). The dispersion curve of the $\mathrm{TM}_{0}$ mode shifts below the PMMA light line at optical energies, which indicates that the fields decrease exponentially from the $\mathrm{Au}-\mathrm{PMMA}$ interface $\left(k_{z}^{2}=\varepsilon_{2}(\omega / c)^{2}-k_{\|}^{2}<0\right)$. Unlike the
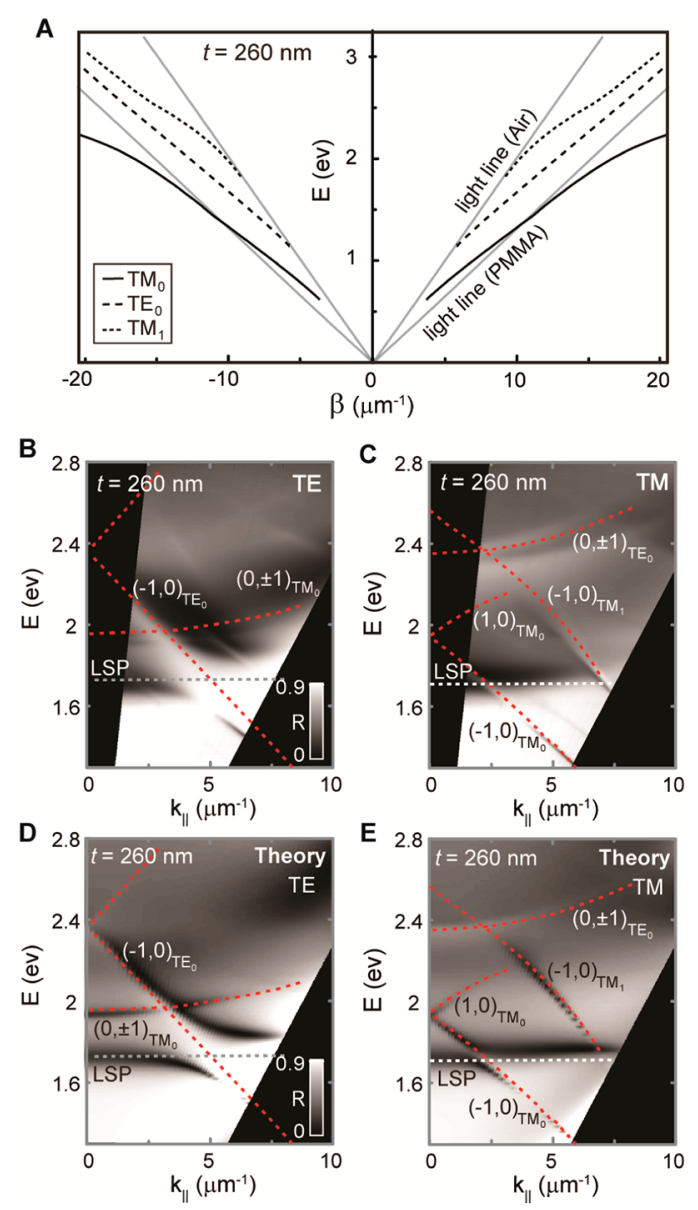

Figure 6. MIM plasmonic nanocavity arrays with thick insulator layers support high-order guided modes. (A) Calculated $E-\beta$ dispersion curves of the three lowest guided modes $\left(\mathrm{TM}_{0}, \mathrm{TM}_{1}, \mathrm{TE}_{1}\right)$ in $\mathrm{Au}$ PMMA-air planar structures with a large PMMA thickness $(t=260$ $\mathrm{nm}) .(\mathrm{B}, \mathrm{C})$ Measured and (D,E) calculated band diagrams of 2D MIM arrays under $(\mathrm{B}, \mathrm{D})$ TE- and $(\mathrm{C}, \mathrm{E}) \mathrm{TM}$-polarized light.

$\mathrm{TM}_{0}$ mode, the curves of the $\mathrm{TE}_{0}$ and the $\mathrm{TM}_{1}$ modes are located between the PMMA light line and air light line; thus, the fields extend uniformly into the PMMA layer but decrease exponentially into air from the air-PMMA interface.

Figure $6 \mathrm{~B}, \mathrm{C}$ shows that the $\mathrm{TE}_{0}$ and $\mathrm{TM}_{0}$ guided modes in MIM plasmonic nanocavity arrays with large $t(\sim 260 \mathrm{~nm})$ exhibit different coupling strengths when they interact with LSPs. Under TE polarization, the $(-1,0) \mathrm{TE}_{0}$ and $(0, \pm 1)$ $\mathrm{TM}_{0}$ guided modes were excited as well as the LSPs of individual NPs $(E \approx 1.82 \mathrm{eV}$ ) (Figure 6B). Interestingly, the $(-1,0) \mathrm{TE}_{0}$ mode exhibited a large Rabi splitting energy of $\sim 0.3 \mathrm{eV}$ (at $k_{\|} \approx 5 \mu \mathrm{m}^{-1}$ ) when coupled to the LSP band, in good agreement with the FDTD-calculated dispersion diagram (Figure 6D). Under TM-polarized light, MIM arrays revealed spectral features corresponding to $(-1,0) \mathrm{TM}_{0},(1,0) \mathrm{TM}_{0}$, $(-1,0) \mathrm{TM}_{1}$, and $(0, \pm 1) \mathrm{TE}_{0}$ guided modes in addition to LSPs (Figure 6C). The anticrossing between the $(-1,0) \mathrm{TM}_{0}$ mode and the LSP was observed at $k_{\|} \approx 2 \mu \mathrm{m}^{-1}$ with a Rabi splitting of $<0.03 \mathrm{eV}$. Compared with the $\mathrm{TE}_{0}$ mode, the optical fields of the $\mathrm{TM}_{0}$ mode were more concentrated at the $\mathrm{Au}-$ PMMA interface instead of extending across the PMMA layer (Supporting Information, Figure S3). Therefore, less spatial overlap of the near-fields between the LSP and $\mathrm{TM}_{0}$ mode results in a smaller difference between the local field 
distributions of the upper and lower branches of the hybridized modes as well as a smaller Rabi splitting energy.

\section{CONCLUSIONS}

In summary, we demonstrated that $2 \mathrm{D}$ arrays of MIM nanocavities can support localized and guided SP modes with different polarization and dispersion properties. Coupling between these localized and guided modes to form new hybrid modes can be characterized by Rabi splitting, which corresponds to slower propagation speeds of optical energy and additional concentration of the localized optical fields. Therefore, 2D MIM nanocavity arrays combine the advantages of optical tunability, strong local field enhancement, and wideangle broadband absorption. Such characteristics could be useful for a wide range of applications, from photovoltaics to efficient light emitters to nonlinear optics.

\section{ASSOCIATED CONTENT}

\section{S Supporting Information}

Measured reflectance spectra versus in-plane wavevector and energy for MIM nanocavity arrays with different thicknesses of the PMMA layer under TE-polarized excitation; measured and FDTD-calculated band diagram of 2D MIM plasmonic nanocavity arrays under TM-polarized excitation and $|E|^{2}, E_{z}$, and $\Phi\left(E_{z}\right)$ distribution maps; and LSP modes showing different anti-crossing behavior with TE and TM guided modes in 2D MIM plasmonic nanocavity arrays $(t=260 \mathrm{~nm})$. This material is available free of charge via the Internet at http://pubs.acs.org.

\section{AUTHOR INFORMATION}

\section{Corresponding Author}

*E-mail: todom@northwestern.edu.

\section{Notes}

The authors declare no competing financial interest.

\section{ACKNOWLEDGMENTS}

This work was supported by the NSF-MRSEC program at the Materials Research Science and Engineering Center at Northwestern University (DMR-1121262) and was also supported as part of the ANSER Center, an Energy Frontier Research Center funded by the U.S. Department of Energy, Office of Science, Office of Basic Energy Sciences (award no. DE-SC0001059). This work made use of the NUANCE Center facilities, which are supported by NSF-MRSEC, NSF-NSEC, and the Keck Foundation.

\section{REFERENCES}

(1) Kelf, T. A.; Sugawara, Y.; Baumberg, J. J.; Abdelsalam, M.; Bartlett, P. N. Plasmonic Band Gaps and Trapped Plasmons on Nanostructured Metal Surfaces. Phys. Rev. Lett. 2005, 95, 116802.

(2) Yang, J. C.; Gao, H. W.; Suh, J. Y.; Zhou, W.; Lee, M. H.; Odom, T. W. Enhanced Optical Transmission Mediated by Localized Plasmons in Anisotropic, Three-Dimensional Nanohole Arrays. Nano Lett. 2010, 10, 3173-3178.

(3) Barnes, W. L.; Dereux, A.; Ebbesen, T. W. Surface Plasmon Subwavelength Optics. Nature 2003, 424, 824-830.

(4) Zhang, X.; Yang, X. D.; Ishikawa, A.; Yin, X. B. Hybrid Photonic Plasmonic Crystal Nanocavities. ACS Nano 2011, 5, 2831-2838.

(5) Maier, S. A. Plasmonics: Fundamentals and Applications; Springer: New York, 2007.

(6) Stewart, M. E.; Mack, N. H.; Malyarchuk, V.; Soares, J. A. N. T.; Lee, T. W.; Gray, S. K.; Nuzzo, R. G.; Rogers, J. A. Quantitative Multispectral Biosensing and 1D Imaging Using Quasi-3D Plasmonic Crystals. Proc. Natl. Acad. Sci. U.S.A. 2006, 103, 17143-17148.
(7) Pala, R. A.; White, J.; Barnard, E.; Liu, J.; Brongersma, M. L. Design of Plasmonic Thin-Film Solar Cells with Broadband Absorption Enhancements. Adv. Mater. 2009, 21, 3504-3509.

(8) Hetterich, J.; Bastian, G.; Gippius, N. A.; Tikbodeev, S. G.; von Plessen, G.; Lemmer, U. Optimized Design of Plasmonic MSM Photodetector. IEEE J. Quantum Electron. 2007, 43, 855-859.

(9) Baumberg, J. J.; Kelf, T. A.; Sugawara, Y.; Cintra, S.; Abdelsalam, M. E.; Bartlett, P. N.; Russell, A. E. Angle-Resolved Surface-Enhanced Raman Scattering on Metallic Nanostructured Plasmonic Crystals. Nano Lett. 2005, 5, 2262-2267.

(10) Wurtz, G. A.; Pollard, R.; Zayats, A. V. Optical Bistability in Nonlinear Surface-Plasmon Polaritonic Crystals. Phys. Rev. Lett. 2006, 97, 057402.

(11) Gao, H. W.; Zhou, W.; Odom, T. W. Plasmonic Crystals: A Platform to Catalog Resonances from Ultraviolet to Near-Infrared Wavelengths in a Plasmonic Library. Adv. Funct. Mater. 2010, 20, 529539.

(12) Linden, S.; Kuhl, J.; Giessen, H. Controlling the Interaction between Light and Gold Nanoparticles: Selective Suppression of Extinction. Phys. Rev. Lett. 2001, 86, 4688-4691.

(13) Christ, A.; Zentgraf, T.; Tikhodeev, S. G.; Gippius, N. A.; Kuhl, J.; Giessen, H. Controlling the Interaction between Localized and Delocalized Surface Plasmon Modes: Experiment and Numerical Calculations. Phys. Rev. B 2006, 74, 155435.

(14) Cai, W. S.; Chettiar, U. K.; Yuan, H. K.; de Silva, V. C.; Kildishev, A. V.; Drachev, V. P.; Shalaev, V. M. Metamagnetics with Rainbow Colors. Opt. Express 2007, 15, 3333-3341.

(15) Christ, A.; Ekinci, Y.; Solak, H. H.; Gippius, N. A.; Tikhodeev, S. G.; Martin, O. J. F. Controlling the Fano Interference in a Plasmonic Lattice. Phys. Rev. B 2007, 76, 201405.

(16) Aydin, K.; Ferry, V. E.; Briggs, R. M.; Atwater, H. A. Broadband Polarization-Independent Resonant Light Absorption Using Ultrathin Plasmonic Super Absorbers. Nat. Commun. 2011, 2.

(17) Ghoshal, A.; Kik, P. G. Theory and Simulation of Surface Plasmon Excitation Using Resonant Metal Nanoparticle Arrays. J. Appl. Phys. 2008, 103, 113111.

(18) Chu, Y. Z.; Crozier, K. B. Experimental Study of the Interaction between Localized and Propagating Surface Plasmons. Opt. Lett. 2009, 34, 244-246.

(19) Chanda, D.; Shigeta, K.; Truong, T.; Lui, E.; Mihi, A.; Schulmerich, M.; Braun, P. V.; Bhargava, R.; Rogers, J. A. Coupling of Plasmonic and Optical Cavity Modes in Quasi-Three-Dimensional Plasmonic Crystals. Nat. Commun. 2011, 2.

(20) Leveque, G.; Martin, O. J. F. Tunable Composite Nanoparticle for Plasmonics. Opt. Lett. 2006, 31, 2750-2752.

(21) Wu, C. H.; Neuner, B.; Shvets, G.; John, J.; Milder, A.; Zollars, B.; Savoy, S. Large-Area Wide-Angle Spectrally Selective Plasmonic Absorber. Phys. Rev. B 2011, 84.

(22) Cattoni, A.; Ghenuche, P.; Haghiri-Gosnet, A. M.; Decanini, D.; Chen, J.; Pelouard, J. L.; Collin, S. $\lambda^{3} / 1000$ Plasmonic Nanocavities for Biosensing Fabricated by Soft UV Nanoimprint Lithography. Nano Lett. 2011, 11, 3557-3563.

(23) Henzie, J.; Lee, J.; Lee, M. H.; Hasan, W.; Odom, T. W. Nanofabrication of Plasmonic Structures. Annu. Rev. Phys. Chem. 2009, $60,147-165$.

(24) Kaminow, I. P.; Mammel, W. L.; Weber, H. P. Metal-Clad Optical-Waveguides - Analytical and Experimental Study. Appl. Opt. 1974, 13, 396-405.

(25) Rashleigh, S. C. 4-Layer Metal-Clad Thin-Film OpticalWaveguides. Opt. Quantum Electron. 1976, 8, 49-60.

(26) Johnson, P. B.; Christy, R. W. Optical-Constants of NobleMetals. Phys. Rev. B 1972, 6, 4370-4379.

(27) Kitson, S. C.; Barnes, W. L.; Bradberry, G. W.; Sambles, J. R. Surface Profile Dependence of Surface Plasmon Band Gaps on Metallic Gratings. J. Appl. Phys. 1996, 79, 7383-7385.

(28) Kitson, S. C.; Barnes, W. L.; Sambles, J. R. Full Photonic Band Gap for Surface Modes in the Visible. Phys. Rev. Lett. 1996, 77, 26702673. 
(29) Nordlander, P.; Prodan, E. Plasmon Hybridization in Nanoparticles near Metallic Surfaces. Nano Lett. 2004, 4, 2209-2213.

(30) Christ, A.; Tikhodeev, S. G.; Gippius, N. A.; Kuhl, J.; Giessen, H. Waveguide-Plasmon Polaritons: Strong Coupling of Photonic and Electronic Resonances in a Metallic Photonic Crystal Slab. Phys. Rev. Lett. 2003, 91, 183901.

(31) Christ, A.; Zentgraf, T.; Kuhl, J.; Tikhodeev, S. G.; Gippius, N. A.; Giessen, H. Optical Properties of Planar Metallic Photonic Crystal Structures: Experiment and Theory. Phys. Rev. B 2004, 70, 125113. 